\title{
Harlem: un folio del archivo (intelectual) de Manuel Zapata Olivella*
}

\author{
Ligia Aldana ${ }^{1}$ \\ SUNY, New Paltz
}

ORCID ID: 0000-0002-6787-8155

Recibido: 17 de diciembre de 2019

Aceptado: 22 de marzo de 2020

\section{RESUMEN}

Este estudio examina el lugar de Harlem, el pos-Harlem Renaissance, en el archivo zapataolivellano. El objetivo es contribuir con los estudios sobre el significado de este legendario espacio negro en el imaginario diaspórico afro, además de determinar la influencia del mismo sobre la narrativa de Manuel Zapata Olivella. Igualmente, este análisis apunta hacia Harlem como espacio que inspira a Zapata Olivella a adoptar decididamente una agenda de resistencia, y subraya la intención del autor de denunciar la marginalidad de Harlem, pos-Renaissance, dentro de la frágil realidad imperialista y supremacista norteamericana.

Palabras clave: Harlem - Manuel Zapata Olivella - Langston Hughes

\begin{abstract}
This study examines the place of Harlem, post-Harlem Renaissance, in the Zapataolivellan archive. The objective is to contribute to studies on the meaning of this legendary black space within the Afro diasporic imaginary, as well as to determine its influence on Manuel Zapata Olivella's narrative. In addition, this analysis points to Harlem as a space that inspires the author to decisively adopt an agenda of resistance, and underscores Manuel Zapata Olivella's intention to denounce Harlem's marginality, post-Renaissance, within the fragile US imperialist and supremacist reality.
\end{abstract}

Keywords: Harlem - Manuel Zapata Olivella - Langston Hughes

\footnotetext{
*Harlem: A Folio from Manuel Zapata Olivella's (Intellectual) Archive

${ }^{1}$ Profesora asociada en español, literatura y cultura latinoamericanas, Department of Languages, Literatures \& Cultures, SUNY New Paltz. Correo: aldanal@newpaltz.edu
} 
So we stand here

On the edge of hell

in Harlem

And look out on the world

And wonder

What we're gonna do

In the face of what

We remember.

(Langston Hughes, "Harlem”2)

Harlem vive su vida. Una vida propia, genuina, de la que no exhibe a nadie ni sus grandezas ni sus miserias... es posible encontrar muchas maravillas, gran parte de la leyenda que lo hiciera famoso.

(Manuel Zapata Olivella, "Harlem olvidado": 101)

En Colombia, la muerte de Langston Hughes en 1967 fue anunciada en un artículo publicado en uno de los periódicos nacionales bajo el título "El legado que deja... Langston Hughes: Risas y lágrimas del negro" (13). ${ }^{3}$ La autora del artículo, Lily Leino, apunta detalles de la vida del poeta, su contribución intelectual, sus ideas y derroteros políticos, sus viajes, y lo define como un intelectual "prodigioso" (13). El final del artículo respalda las declaraciones de los críticos quienes afirman que "ningún poeta de nuestros días ha captado la calidad sincopada del jazz mejor que Hughes" (17). El anuncio de la muerte del poeta sin duda subraya la relación intelectual y conexión humana que este último compartía con América Latina, y sirve de recordatorio de la causa de los afrodescendientes a la que el poeta dedicó su vida y a la que Manuel Zapata Olivella consagraría la suya, convirtiéndose ambos en abanderados de la causa afro ${ }^{4}$ en las Américas.

Durante la década de los sesenta, cuando tanto en Colombia como en el resto de América Latina los escritores caminaban ya el sendero del realismo mágico, Manuel Zapata Olivella reclama el realismo como el vehículo que mejor encajaba la herencia africana en el canon colombiano. ${ }^{5} \mathrm{La}$ producción intelectual de Hughes en poesía, narrativa, teatro, y periodismo, para nombrar solo algunos de los géneros de sus publicaciones, marcaría la narrativa de Zapata Olivella, profundamente. Pero es el espacio de Harlem, el hogar ideológico e intelectual de Hughes, quien no deja de cuestionar los privilegios de los literati, el que evidencia para Zapata Olivella una socio-historicidad que le sirve de cuna cultural, política e identitaria y le presta una perspectiva sobre el lugar del

\footnotetext{
${ }^{2}$ The Poetry Foundation https://www.poetryfoundation.org/poems/46548/harlem

${ }^{3}$ La referencia de este artículo está incompleta, ya que no contiene el nombre de la publicación, ni la fecha exacta de ella. La fuente proviene de las Manuel Zapata Olivella's Collections de la Jean and George Heard Library de Vanderbilt University, Nashville, Tennessee. Por opción propia y para examinar a fondo el pensamiento del autor desde las primeras notas que luego hicieron parte de He visto la noche y Hotel de vagabundos, escogí utilizar las copias de los archivos de la citada colección como textos primarios. Entre los tachones y re-escritura de secciones es fácil seguir la línea ideológica zapataolivellana, más que en los ya editados y publicados textos para el público general.

${ }^{4}$ En este ensayo, me tomo la libertad de utilizar los términos "afro" y "afrodescendientes" a través del texto, aún cuando discuto elementos que cronológicamente no concuerdan con aquéllos. Lo hago para enfatizar lo logrado por los afrodescendientes, y con toda consciencia que tanto Hughes como Zapata Olivella utilizaban en término en boga; negro. Ver Aldana (2006) para examinar los comentarios de Zapata Olivella sobre su propia evolución en cuanto al uso de la palabra "afro." Aunque éste es sintácticamente un sufijo, "afro" ha pasado a ser un sustantivo que define identidad, etnicidad, y herencia; en sí, africanitud. Me adhiero a este uso, y afirmo así el valor y poder de su enunciación.

${ }^{5}$ Ver The Culture of Fiction in the Works of Manuel Zapata Olivella de Yvonne Captain-Hidalgo. Este es el estudio más completo sobre el uso del realismo como parte de la propuesta del trabajo de Zapata Olivella.
} 
entonces sujeto negro, ahora sujeto afro, que reclama sus derechos humanos e intelectuales. Este análisis no se concentra meramente en la relación entre Hughes y Zapata Olivella, a pesar de que hay necesidad de anotar elementos de sus lazos literarios e ideológicos en determinados momentos textuales. El objetivo es indagar también sobre el lugar de Harlem en el archivo zapataolivellano, para contribuir con unas cuantas ideas a la discusión sobre este importante lugar de la diáspora africana. No es el foco tampoco el Harlem sublimado por un renacimiento intelectual y político que fue simultáneamente producto consumido por los estadounidenses caucásicos liberales quienes adoptaban protégés negros y causas para ellos novedosas. El eje es el Harlem que encuentra Zapata Olivella en el periodo pos-Harlem Renaissance, cuya realidad lo lleva a reconsiderar el verdadero significado de este espacio negro como parte del imaginario diaspórico afro, ${ }^{6}$ y que pasa a nutrir su narrativa y a darle la profunda aura de resistencia que a tantos ha inspirado. Imposible no mencionar la figura del intelectual orgánico que emerge en la imagen de Hughes y que Zapata Olivella encarna a través de su vida. Sin embargo, este ensayo intenta explorar más allá de la relación de Zapata Olivella y Hughes para enfocarse en Harlem como espacio influyente en la producción textual del autor de forma específica. ${ }^{7}$ Al mismo tiempo, vale reconocer una cierta intención de parte de Zapata Olivella por subrayar la marginalidad de Harlem pos-Renaissance dentro de una frágil realidad imperialista y supremacista norteamericana.

\section{Manuel Zapata Olivella y Langston Hughes: Intelectuales pan-afroamericanos}

La conexión Langston Hughes-Manuel Zapata Olivella ya ha sido visitada y discutida por críticos que subrayan la importancia de la consolidación de una continuidad intelectual pan-afroamericana. Dice Richard Jackson ${ }^{8}$ en 1981, que

Hughes [is] not only one of the foremost Hispanists in the United States, but also one whose own black consciousness shows distinct affinities with black authors writing in Spanish. Langston Hughes shares a common heritage of slavery, racism, and oppression. His work has both influenced and been shaped by others who share the same heritage. (89) ${ }^{9}$

Jackson demuestra la veracidad de su afirmación al mencionar a la uruguaya Pilar Barrios, al cubano Nicolás Guillén, a Zapata Olivella y otros, quienes expresan su admiración por Hughes en sus poemas y textos. Laurence Prescott, otro trascendente crítico, estudioso de la literatura afrolatinoamericana y en especial de las letras afrocolombianas, afirma en su introducción a la edición más reciente de He visto la noche (1969), que Zapata Olivella se había informado debidamente "sobre Harlem y la situación de los negros, utilizando fuentes impresas como The Big Sea (1940),

\footnotetext{
${ }^{6}$ En ese momento histórico, Zapata Olivella hubiera dicho diáspora africana, imaginario negro. Escojo enfatizar un continuo diaspórico afro para hacer parte del esfuerzo aún vigente de afirmar este hilo diaspórico a nivel hemisférico, y recalcar el lugar de Colombia en el mismo.

${ }^{7}$ Aclaro aquí que otra versión de este ensayo analiza el paralelo entre Chambacú como texto y espacio, y Harlem, parte que no incluyo en esta sección.

${ }^{8}$ Richard Jackson es uno de los pioneros en el estudio de lo que ahora se reconoce como literatura afrolatinoamericana, el cual fue en gran parte producto del movimiento panafroamericano, el movimiento de la negritudes y la influencia del movimiento afroamericano por los derechos civiles. 9 "Hughes no es solamente uno de los principales hispanistas de Estados Unidos, sino también uno de los intelectuales cuya conciencia afroamericana muestra una afinidad particular con autores negros que escriben en español. Langston Hughes comparte una herencia común de esclavitud, racismo y opresión. Su trabajo ha servido tanto como influencia como de fuente de formación por otros quienes comparten la misma herencia" (Traducción mía).
} 
la primera autobiografía de Langston Hughes" (237). ${ }^{10}$ En publicaciones posteriores, Prescott explora la correspondencia que Hughes y Zapata Olivella intercambian a través de los años, la cual a pesar de ser limitada, subraya los puntos de contacto que existen entre los temas de sus obras y sus libros de viaje. ${ }^{11}$ La relación que cuidadosamente Edward J. Mullen devela en Langston Hughes in the Hispanic World and Haiti (1977) entre el escritor afroestadounidense ${ }^{12}$ y numerosos poetas afro y latinoamericanos en general de la época es retomada por Laurence Prescott para explorar en particular la amistad e influencia de Hughes sobre Manuel Zapata Olivella. En una época de inmensa dificultad para la comunicación entre intelectuales a través de fronteras y los obstáculos que la circulación de textos presentaba, Zapata Olivella y Hughes intercambiaron varias cartas y publicaciones que los mantuvieron conectados a pesar de la distancia que los separaba.

Es relativamente fácil entender cómo "El Poeta de los Negros" y "El Decano de las Letras Negras" se convierte en inspiración para los entonces poco conocidos escritores afrolatinoamericanos (Mullen: 15-46), ya que su importancia estaba atada a "the influence of Hispanic motifs in his own work [...] from a complex set of factors, both historical and aesthetic" (Mullen: 15-46). ${ }^{13}$ Otra obvia y especial conexión entre Hughes y Zapata Olivella se encuentra en sus vidas de autodefinidos "vagabundos" e intelectuales negros. De hecho, Francisco Javier Flóres Bolívar examina el diálogo diaspórico entre el Harlem Renaissance y el activismo afrocolombiano al exaltar la significativa dimensión política del movimiento. Más recientemente, George Palacios ofrece un análisis a fondo sobre la relación entre Langston Hughes y Zapata Olivella, y el papel de Harlem como un elemento "explicativo de la situación del sujeto racializado en los contextos sociales, políticos y culturales en el hemisferio (94). ${ }^{14}$ En la mayoría de los estudios antes mencionados, Hughes y Harlem son sinónimos. Cuando Zapata Olivella viaja a lo largo del continente hacia el norte hasta llegar a Harlem, su intención era conocer tanto el lugar como a Hughes.

Ahora, a pesar de los estudios mencionados anteriormente, y de muchos otros no incluidos en este estudio, la propuesta de este ensayo amerita una justa pregunta: ¿Qué aporta el Harlem pos-Renacimiento, cuyo momento cúspide como espacio cultural, intelectual y artístico ya estaba en su cenit, al pensamiento zapataolivellano? Para el escritor afrocolombiano, quien había "vagabundeado" sin dinero y con ansias de ver el mundo y Harlem, la conexión era clara: la circunscripción

\footnotetext{
${ }^{10}$ En el pie de página 11 incluido en estas líneas, Prescott explica que una traducción al español de la autobiografía de Hughes había sido publicada en 1944 bajo el título El inmenso mar (237).

${ }^{11}$ Ver "We, Too, Are America: Langston Hughes in Colombia" y "Brother To Brother: The Friendship And Literary Correspondence Of Manuel Zapata Olivella And Langston Hughes."

${ }^{12}$ Anoto que utilizo el término afroamericano cuando las citas lo contienen, o cuando el marco histórico lo requiere. Sin embargo, prefiero emplear el vocablo identitario "afroestadounidense" como forma de crear conciencia sobre la apropiación de una referencia continental y hemisférica por parte de un país. Respeto el hecho que establecer el uso de "afroamericano" era importante, y aún lo es para muchos descendientes de africanos en EEUU para reclamar su derecho a ser partícipes completos en la sociedad que ayudaron a construir. No obstante, este término subraya una complicidad con el proyecto capitalista y opresor de los EEUU. El camino es largo, pero ya circula la idea; ver Lomas (2009) como ejemplo de una propuesta de cambio por medio del uso mismo de una palabra diferente para referirse a una tradición literaria estadounidense. En medios de comunicación ya había comentarios desde los años 80: ver Saffire (1986).

13 "La influencia de los motifs hispanos en su propio trabajo [...] a partir de un complejo conjunto de factores, tanto históricos como estéticos". (Traducción mía).

${ }^{14}$ Ver Identidades políticas en tiempos de afrodescendencia: auto-identificación, ancestralidad, visibilidad y derechos, de Silvia Valero y Alejandro Campos García (2015) para examinar conceptos contemporáneos de identidad afro en América Latina.
} 
de una población negra dentro de un espacio a razón de su herencia étnica, y su alienación dentro de la agenda nacional y urbana contenida en un continuo capitalista y racista y cómo, precisamente desde esa marginalidad, emergen una consciencia política negra y una creatividad desbordadas.

En el momento de su viaje a Harlem, Zapata Olivella estaba en su fase de búsqueda personal e intelectual. De hecho, las circunstancias de los afrodescendientes en Colombia inspiran en él la necesidad de examinar por sí mismo el legendario vecindario estadounidense, un "corral" como los muchos pueblos y vecindarios latinoamericanos negros, olvidados, donde el activismo político no había alcanzado lo que es hoy, y en los que los intelectuales negros se sacrificaban por la supervivencia diaria. El deseo de Zapata Olivella radicaba en experimentar directamente lo que había sido convertido en un espacio negro propio, a pesar de haber sido reducido nuevamente a los márgenes después de su florecimiento. Este deseo nace de su aspiración por forjar las estrategias y militancia necesarias para conquistar en Colombia, y en el continuum americano, lo propio negro/ afro, colombiano, diaspórico y hemisférico.

\section{El Harlem de los años veinte}

Si bien el Harlem Renaissance produce un inmenso corpus de artefactos culturales y literarios en diversos géneros, es la emergencia del intelectual orgánico negro lo que abre la puerta al futuro y a la esperanza para la comunidad y el EEUU jimcrowniano. El New Negro es articulado dentro de sus propias coordenadas como respuesta a las fuerzas que lo marginan y se yergue como figura de cambio que resiste las políticas de lugar y raza en el contexto diaspórico negro estadounidense; una imagen y posibilidad aterradoras para la clase dominante de la época, firmemente enraizada en su cosmovisión eurocéntrica y supremacista.

Innumerables son las fuentes y críticos que ensalzan el apogeo del Harlem Renaissance y rinden homenaje al sinnúmero de intelectuales y artistas negros que le dieron paso. Los elementos que originaron el ascenso del niggeratti, como llamaba el excéntrico Wallace Thurman a los intelectuales que se distinguieron durante la época de los años veinte y treinta en Harlem -Jean Toomer, Claude McKay, Countée Cullen, Zora Neale Hurston, Alan Locke, y cantantes y bailarinas como Florence Mills and Josephine Baker entre muchos otros,- tiene todo que ver con la migración de afroestadounidenses del sur al norte del país después de la Primera Guerra Mundial. ${ }^{15}$ Los cambios demográficos en el área que vendría a convertirse en el centro de la producción cultural afroestadounidense fueron creados por la inicial decadencia en el mercado de bienes raíces neoyorquino y la necesidad de los propietarios, inmigrantes europeos en su mayoría, de alquilar sus dilapidados edificios a inquilinos negros a bajos precios. ${ }^{16}$ A manera de espacio de reunión y de protección para

\footnotetext{
${ }^{15}$ Para examinar la trayectoria e historia de Harlem, ver Black Manhattan de James Weldon Johnson y Harlem, the Making of a Ghetto: Negro New York, 1890-1930 de Gilbert Osofsky; para explorar el Harlem Rennaissance ver The Harlem Renaissance: A Brief History with Documents de Jeffrey B. Ferguson.

${ }^{16}$ Ver Johnson and Osofky.
} 
las familias e individuos negros en el no menos hostil ambiente de La Gran Manzana de principios del siglo XX, Harlem se convierte en un oasis negro donde la vida de sus habitantes, quienes para los años veinte sumaban casi 400,000 y eran predominantemente negros, encontraron una oportunidad para expresarse culturalmente y crear comunidad.

In 1920 [dice Jeffrey B. Ferguson] Harlem stretched six blocks from Lexington Avenue on the east side to St. Nicholas Avenue on the west. From north to south it covered about twenty blocks, or approximately one mile, between $125^{\text {th }}$ and $145^{\text {th }}$ streets. In this area, which expanded fifteen blocks to the south and ten blocks to the north by 1930 and grew in population from about 92,000 in 1910 to 328,000 in 1930, one could find the main headquarters of many significant organizations, including the UNIA [The Universal Negro Improvement Association] and the National Urban League. Here were the homes and offices of many civil rights leaders of national importance, including W.E.B. Du Bois, James Weldon Johnson, and A. Philip Randolph. The major journals connected to important civil rights organizations, such as Opportunity... and Negro World...had offices here. The Silent March originated in Harlem; Marcus Garvey's colorful parades always occurred here; and the victorious $369^{\text {th }}$ Regiment called it home. $(8)^{17}$

Estos significativos elementos convirtieron la circunscrita área en un foco político, cultural, social y étnico que atraía a negros no solo del sur de Estados Unidos, sino también de todo el mundo: "Immigrants from the West Indies and Africa mixed freely with migrants from the South and with black New Yorkers whose families had resided in the city since the colonial period" (8). ${ }^{18}$ Para muchos, el auge de Harlem era una realidad, especialmente para los intelectuales quienes adquirieron fama y éxito, y para los afroestadounidenses adinerados de sociedad que se codeaban con los blancos ricos que frecuentaban los famosos clubes solo para blancos donde "the strangers were given the best ringside tables to sit and stare at the Negro customers-like amusing animals in a zoo" (Hughes The Big Sea in The Langston Hughes Reader: 369). ${ }^{19}$ La intención de Langston Hughes en estas palabras previas es establecer que el Harlem que estaba en vogue en los años veinte y treinta no era el mismo para todos sus habitantes. "The ordinary Negroes hadn't Heard of the Negro Renaissance. And if they had, it hadn't raised their wages any" (371). ${ }^{20}$ Para los trabajadores negros, a los que Hughes llama Harlemites, la realidad de Harlem era igual a las otras comunidades segregadas alrededor de Estados Unidos: un espacio pobre, de limitados recursos, donde la vida se

\footnotetext{
${ }^{17}$ En 1920, [dice Jeffrey B. Ferguson,] Harlem se extendía seis cuadras desde Lexington Avenue en el lado este hasta St. Nicholas Avenue en el oeste. De norte a sur cubría unas veinte cuadras, o aproximadamente una milla, entre las calles 125 y 145. En esta área, la cual se expandió quince cuadras hacia el sur y diez cuadras hacia el norte en 1930 y cuya población aumentó de aproximadamente 92,000 personas en 1910 a 328,000 en 1930, uno podría encontrar la sede principal de muchas organizaciones importantes, incluidas la UNIA y el National Urban League. Aquí estaban las casas y oficinas de muchos líderes de derechos civiles de importancia nacional, incluso W.E.B. Du Bois, James Weldon Johnson y A. Philip Randolph. Las principales revistas relacionadas con importantes organizaciones de derechos civiles, como Opportunity [...] y Negro World [...] tenían oficinas ahí. La marcha silenciosa se originó en Harlem; los coloridos desfiles de Marcus Garvey siempre ocurrían aquí; y el victorioso Regimiento 369 lo llamó su hogar". (Traducción mía).

18 "Los inmigrantes de las Indias Occidentales y África se mezclaron libremente con los migrantes del Sur y con los neoyorquinos negros cuyas familias habían residido en la ciudad desde el período colonial". (Traducción mía).

19 "A los desconocidos se les dieron las mejores mesas junto al ring para sentarse y mirar a los clientes negros como entretenidos animales en un zoológico”.(Traducción mía).

20 "Los negros comunes no habían oído hablar del Renacimiento negro. Y si lo hubieran hecho, no habría aumentado sus salarios". (Traducción mía).
} 
sobrevivía, a pesar del jazz y la poesía, o debido a estos. Los rent parties, fiestas para recoger fondos para pagar el alquiler, eran las fiestas preferidas de Hughes donde él "met ladies' maids and truck drivers, laundry workers and shoe shine boys, seamstresses and porters" (374). ${ }^{21}$ Evidentemente, Hughes encuentra su ambiente no entre el Harlem Literati, sino entre la clase trabajadora que vivía en el Harlem real, no en el Harlem idealizado. "I don't know what made any Negroesthink that -except that they were mostly intellectuals doing the thinking" $(371)^{22}$, dice Hughes, al referirse a la razón por la cual Harlem era percibido como un centro de intelectuales negros eruditos. La actitud de Hughes hacia los intelectuales y los harlemnianos adinerados propietarios de bienes raíces y negocios es parecida a la que expresa durante su estadía en Washington, D.C., donde "the better class" -la mejor clase- (Hughes, 2002: 206) discriminaba a los negros de clase obrera: "They were on the whole as unbearable and snobbish a group of people as I have ever come in contact with anywhere. They lived in comfortable homes [...] but seemed to me altogether lacking in real culture, kindness, or good common sense" (206-207). ${ }^{23}$ Pese a sus quejas, Harlem, en todo su apogeo en los años veinte y treinta, y a pesar de su persistente pobreza, era para Hughes un lugar afín a su sensibilidad política e intelectual, y a su identidad étnica. Hughes hubiera podido quedarse en Washington, o en una de las muchas ciudades que visitaba durante su círculo de conferencias y lecturas de poesía. Por el contrario, Harlem se convirtió en su hogar hasta su muerte. ${ }^{24}$

\section{Manuel Zapata Olivella en Harlem}

En 1947, cuando Zapata Olivella llega a Harlem con la ilusión de experimentar la realidad del mito que rodeaba el lugar, la gloria del vecindario ya había desaparecido. Después de la Gran Depresión de 1929, "the crash [...] sent Negroes, White folks and all rolling down the hill towards the Works Progress Administration" (369). ${ }^{25}$ Para ese entonces, "[ya] Harlem no [era] el Harlem de ayer" (Zapata Olivella, 1948: 97), una mina de ideas y un reverbero de poesía, teatro, música y creatividad, y la Meca de los Negros, como se le llamaba. En su crónica, "Harlem olvidado," Zapata Olivella cita artículos publicados en la prensa neoyorquina que denuncian las condiciones de insalubridad de las tiendas y mercados del barrio: "En una de las tiendas el dueño permitía que un gato estuviera sentado sobre los pedazos de carne para la venta. Muchos hoyos de ratas eran visibles, y la mayoría de ellos estaban habitados [...] El espectáculo que ofrece el barrio de hoy es terrible" (98). Con toda certidumbre, estos comentarios están dirigidos a cuestionar la supremacía norteamericana contenida en el discurso oficial estadounidense que señala el "atraso" de los "otros" pueblos americanos.

Pero en medio de ese Harlem de los cuarenta, Zapata Olivella recibe el mejor de los regalos después de su largo peregrinaje por América: conoce a Langston Hughes, quien lo rescata del hambre

\footnotetext{
21 "Conocí a sirvientas y camioneras, trabajadoras de lavandería y limpiabotas, costureras y mozos” (Traducción mía).

${ }^{22}$ No sé qué hizo a los negros pensar eso -excepto que eran en su mayoría los intelectuales quienes pensaban”. (Traducción mía).

23 "En general, eran tan insoportables y presumidas como el peor grupo de personas con las que alguna vez hubiera estado en contacto. Vivían en hogares cómodos, [...] pero me parecían completamente carentes de cultura real, amabilidad o buen sentido común”. (Traducción mía).

${ }^{24}$ Hughes murió en la Stuyvesant Polyclinic situada en la 2da. Avenida, Manhattan, Ciudad de Nueva York. Sus cenizas están enterradas bajo el piso de la antesala del teatro epónimo, sobre el cual está escrito uno de los famosos poetas del autor, en el Arthur Schomburg Center for Research in Black Culture en Harlem.

25 "la Depresión [...] envió a negros, blancos y todos rodando cuesta abajo hacia el Works Progress Administration”. (traducción mía).
} 
y el frío, le ofrece refugio en su apartamento, y le cede su propia cama. En notas que expresan su inmensa gratitud por la generosidad de Hughes, Zapata Olivella habla de su sorpresa al ver que

Langston [le] cedió su cama para refugiarse en la biblioteca. No era un acto de cortesía, deseaba brindar al hermano golpeado por el hambre y los prejuicios raciales que él también, en su vida de marinero por los puertos de África, los bares de Europa y los barrios segregados de los Estados Unidos, había experimentado las mismas inclemencias. (Zapata Olivella notas cuaderno Norma 41$)^{26}$

Dentro de esta afinidad y cercanía intelectual y filial, Zapata Olivella ve a Hughes como una "presencia revitalizadora. El fuego de sus poemas depuraba [sus] confusiones, iluminaba [su] extirpe de esclavo rebelde, las frustraciones de estudiante pobre, [su] ideal de escritor" (45). Esta realización repentina en el pequeño apartamento de Hughes hace que Zapata Olivella decida regresar a Colombia, a la universidad. Reconoce que "[e]l título de médico no significaba tan solo el derecho de ejercer una profesión: sería un arma más a la lucha por desalienar a [su] raza, a [su] pueblo, a [su] familia, a [sí] mismo" (45). Sin duda alguna, los senderos que Langston Hughes y Manuel Zapata Olivella recorrieron por separado los llevaron a encontrar experiencias comunes en Harlem que les permitieron a los dos, y especialmente a éste último, retomar la batalla por la liberación de los negros en América, cada uno con su propio instrumento, el cual luego sería el mismo: una literatura centrada en la denuncia de la represión de los negros y desposeídos de América.

En "Langston Hughes o el valor de la poesía,"27 Zapata Olivella dilucida cuidadosamente las ideas de "El poeta mulato" (458), y subraya las limitaciones que enfrenta el negro en general en cuanto a ocupaciones:

A nosotros los negros solo se nos ocupa como sirvientes, limpiabotas u obreros aunque tengamos títulos universitarios. Si tú sabes de un negro médico, ingeniero o abogado que trabaje en alguna oficina estatal, puedes estar seguro que cualquiera que sea su rango, sus colegas blancos lo tratarán como a un barrendero. (458)

Estas son experiencias ya vividas por Manuel Zapata Olivella en su peregrinaje y algunas en Colombia. Sin embargo, escucharlas de boca de Hughes, insta a Zapata Olivella a repensar sus derroteros, reclamar su profesión y a volver a Colombia para hacerle frente al racismo y la discriminación que comparte con sus coterráneos afro.

\footnotetext{
${ }^{26}$ Cuaderno marca Norma contenido en la Caja 47 de la colección Manuel Zapata Olivella Collections de Vanderbilt University Special Collections \& Archives, Nashville, Tennessee . Este pequeño cuaderno es parte de las decenas de cuadernos en los que Zapata Olivella escribía a mano sus historias, apuntes para libros, conferencias, etc. Están numerados a mano y escritos en ambas direcciones, de la primera a la última página y de la última a la primera, para utilizar hasta el máximo el papel. Los cuadernos, como todas las otras fuentes primarias de Zapata Olivella citadas en este capítulo, son parte de dicha colección.

${ }^{27}$ Este texto se encuentra en la Caja 47 de colección Manuel Zapata Olivella Collections de Vanderbilt University Special Collections \& Archives, Nashville, Tennessee. Es parte de un volumen numerado a mano y escrito a máquina que contiene numerosos textos algunos ya publicados en periódicos, en la Biblioteca [virtual] de Literatura Afrocolombiana o en otras publicaciones, o aún inéditos. No tengo ningún récord que indique que este texto fue publicado por Zapata Olivella, pero es probable que lo haya sido, ya que mucho del poco sustento que consiguió durante su viaje por América venía de las crónicas sobre sus experiencias. Muchas fueron publicadas en México y otras en la revista Sábado, ya descontinuada.
} 


\section{Espacio y negritud: Harlem y Colombia negra, los corralitos de los negros marginados}

Con toda certeza, a su regreso a Colombia, Zapata Olivella puede identificar sin dificultad los contrapuntos de la miseria y la marginalidad que los desposeídos de la tierra y en particular los negros descendientes de africanos esclavizados en América viven en común. Entre Harlem y la Colombia negra había una alienación compartida que cercaba a los habitantes negros en cada espacio; el común ciudadano negro, es obligado a mirar hacia afuera, como cuando se mira la inasequible mercancía a través de las elegantes vitrinas de la Quinta Avenida de Nueva York o de las tiendas de turistas del Centro Histórico de Cartagena. Zapata Olivella comienza así a conectar los puntos del mapa afrodiásporico por medio de los afrodescendientes representados en Harlem después de la Segunda Guerra Mundial, quienes se enfrentan entre sí para pelear por los pocos recursos que el corral harlemniano ofrecía. Zapata Olivella ve en estos hermanos afro de Harlem las mismas batallas que se batían en las costas de Colombia, Chambacú, y todas las regiones ribereñas negras.

Todo lo anterior es necesario para situarse en Harlem y reiterar su importancia, y es igualmente relevante para examinar por qué ese legendario lugar convocaba a Zapata Olivella, hasta conseguir llegar a él. Es, sin duda, una cuestión de "lugar." De encontrar, visitar, examinar y experimentar los espacios negros de la diáspora para unir los puntos en el mapa histórico de la presencia africana en este hemisferio y poder así insertarla en la geografía de sus propios textos. Se hizo evidente para Zapata Olivella durante el viaje hasta llegar a Harlem que los negros del continente, cuya presencia había fortalecido las culturas y la demografía de las Américas, desplegaban la formación de lo que K. A. Appiah denomina comunidades sociohistóricas en su artículo "The Uncompleted Argument: Du Bois and the Illusion of Race," como una estrategia de supervivencia, noción que rescata de los estudios sobre raza de W. B Du Bois. En este estudio, amplío el concepto de Appiah para resaltar la percepción de Zapata Olivella del espacio de Harlem como un espacio creativo de resistencia, que afirma la relación entre lugar y negritud. Esta última subraya la estrecha correspondencia entre lo sociocultural y lo político en los textos de Zapata Olivella, como se ve en su obra de teatro Hotel de vagabundos, situada dentro del marco de los márgenes de la sociedad estadounidense y neoyorquina en plena crisis económica durante la Segunda Guerra Mundial. La circunscripción de una larga lista de personajes tipológicos: refugiados, veteranos de guerra, homosexuales negros, drogadictos, al hotelucho donde luchan por mantenerse vivos, contiene esa aura harlemniana de periferia y abandono que encuentra Zapata Olivella, y que está preñada de un descontento listo para explotar. De hecho, el ambiente de desesperanza, racismo y doom de Hotel de vagabundos denuncia " $[\mathrm{e}] 1$ renacimiento del racismo después de la Segunda Guerra Mundial [...] Mientras miles de negros se sacrificaron en Europa combatiendo contra el exterminio de los judíos, en Atlanta, Mississippi, Virginia, Washington y el propio New York se linchaban negros" ("La República Negra" Manuel Zapata Olivella's Papers Collection Caja 47, Vol. II, 462). El mismo Zapata Olivella "había convivido con hermanos de raza a quienes la sociedad norteamericana mantiene cercados en los campamentos de miseria. En el Mills's Hotel, en pleno corazón de Manhattan, conocí a los miembros más envilecidos en la familia extensa de mis hermanos negros" (463-464). Y fue Langston Hughes quien, con su crítica a los privilegios de los intelectuales de Harlem y la ideología 
supremacista estadounidense que Zapata Olivella experimentó en carne propia, corroboró para el autor el alcance cruel del racismo y lo ayudó a examinar la realidad del Mills's Hotel desde nuevas perspectivas críticas que lo llevarían sin duda a escribir Hotel de Vagabundos: "El poeta mulato Langston Hughes, marinero de los ríos y mares de la discriminación racial de su país, terminó por borrar la ingenuidad de mis ojos acostumbrados a ver el mundo sin recelos" (458). Esto de ninguna manera significa que Zapata Olivella hubiera en algún momento creído que la discriminación no existía en su nativa Colombia o alrededor del mundo. Por el contrario, Zapata Olivella había vivido su propia marginalidad como profesional e intelectual negro. A pesar de ello, reconoce el autor que, hasta entonces, su inclinación seguía las coordenadas de la desigualdad social, pero no había alcanzado una consciencia racial. Pensaba, le dice a Langston Hughes en una de sus conversaciones, que

la posición del negro suramericano es diferente. Tiene conciencia social, pero no racial. Hemos tenido poetas, médicos, jurisconsultos, en fin, se han distinguido en todas las disciplinas del espíritu, pero solo oyendo una vocación, tal vez con sentimiento patriótico, pero nunca por honor a la raza. (Zapata Olivella, 23 de agosto de 1947, 12 cit. en Palacios, 2020: 97)

Las palabras de Zapata Olivella demuestran el proceso de concientización al que Hughes contribuye al señalarle al compadre vagabundo la realidad norteamericana que el Harlem de los 40 ilustra, pos-renacimiento. Zapata Olivella aprende así a ver a Harlem a manera de lente a través del cual las realidades y espacios negros colombianos podían ser revisitados, interpretados y re-escritos. Aún su propia identidad como intelectual negro en busca de su espacio en la ciudad letrada colombiana comienza a orientarse a otros derroteros textuales hasta llegar a Changó, el gran putas, el cual es de verdad un regreso al comienzo, al origen, al muntu africano.

La búsqueda de los innumerables intelectuales negros que llegaron a Harlem, la Capital Negra, es una cuestión de autoría e identidad. De encontrar el espacio o espacios donde anclar una voz propia. Lo necesitaba Zapata Olivella, porque es ese espacio del intelectual orgánico negro harlemniano el que lo impulsa a interpretar el universo negro de las Américas más allá de la marginalidad y la alienación, lo que se traduce a una visión negra liberadora, rebelde, pan-afroamericana. Los finales de Chambacú, Hotel de vagabundos, y los textos completos de Levántate mulato! y Changó, el gran putas, además de los numerosos artículos escritos imbuidos de un tono de lucha y esfuerzo concientizador son evidencia del efecto que Harlem, el Harlem mítico vivido a través de Langston Hughes y la obra de los intelectuales negros de la Meca Negra, tuviera sobre Zapata Olivella. Es imposible que Zapata Olivella olvidara a Marcus Garvey, un ícono del Harlem militante, y su participación en la Second International Convention of Negros, la cual con todo su problemático uso de la retórica democrática estadounidense (Ferguson, 2008: 43), continuaba su reclamo de "Africa for the Africans," África para los africanos. Se entera también Zapata Olivella 
de la consigna de luchar por la formación de una República Negra Independiente en el Sur de los Estados Unidos. La idea venía discutiéndose desde que Marcus Garvey proclamara la Nación Negra constituida por los cuatrocientos millones de negros regados en el mundo. ("La República Negra” Manuel Zapata Olivella’s Papers Collection Caja 47, Vol. II, 462-463)

Estos elementos trazaron para Zapata Olivella en los años cuarenta en Harlem una ruta a seguir en su obra y vida intelectual. Independientemente de las más míseras escenas y eventos descritos en textos como los mencionados anteriormente, Zapata Olivella se esfuerza por ofrecer una dimensión de resistencia y enaltecimiento del poder de los marginados, de los Wretched of the Earth, como los define Franz Fanon. Bien lo dice Zapata Olivella: "pese al odio de las razas, se está gestando un nuevo y más profundo mestizaje que el acumulado en mi etnia caribeña" (468).

Después de su visita a Harlem, su visión para la liberación de los desposeídos de la tierra se convierte en una misión. Su meta es contribuir decididamente y más que nunca a esa lucha pan-afroamericana y global contra las fuerzas que oprimen al negro, al indio, a los pobres y marginados del mundo: "Por vez primera podía contemplar claramente la orilla buscada en cuatro años de oscuridades: un puesto de combate en mi propio país" (468). El deseo que hasta su llegada a Harlem había motivado a Zapata Olivella se convierte en un urgente llamado a la resistencia. Como el Harlem rebelde que "had entirely rid itself of the sense of apology for its existence" (Johnson, 1968: 231) ${ }^{28}$ y recogió a "thousands of Negros [who began] pouring" $(153)^{29}$ en el vecindario, la América rebelde clamaba a Zapata Olivella como líder y voz de los ahora reconocidos como afro. La coyuntura histórica de Cartagena, Palenque y de la costa en general, bajo los nuevos parámetros multiculturalistas y el poder afro en Colombia exhibe los mismos rasgos del proceso de exploración, organización y articulación de los afroamericanos en los EEUU, dentro del cual Harlem fue central. ${ }^{30}$

"Negritud y liberación," un texto escrito en el marco del Diálogo de la Negritud y la América Latina en Dakar, Senegal, organizado por el ilustre Léopold Sédar Senghor, líder del movimiento de la négritude, establece de hecho las amplias conexiones que Zapata Olivella identifica entre las coordenadas de la realidad afro en el hemisferio americano y su "africanitud" (Caja 47, Vol. III, 775). Menciona Zapata Olivella cómo en "América la palabra negritud tiene sus propias resonancias: negro, indio, razas pigmentadas e impuras, 'silver roll', black, nigger, etc." (769), palabras que muestran una clara comprensión del significado de estos términos en la América de habla inglesa y que crean un paralelo explícito entre la realidad norteamericana de la época y la de la otra América. Ese espacio norteamericano en su apogeo jimcrowniano, razón por la cual Hughes se radica en Harlem, produce

\footnotetext{
28 "se había librado por completo del sentido de disculpa por su existencia". (Traducción mía).

29 "Miles de Negros [que comenzaron] a llegar". (Traducción mía).

${ }^{30}$ Esta afirmación merita explicación, lo cual me es imposible ofrecer dentro de este ensayo. Sin embargo, adelanto que, si bien la nueva constitución colombiana ofrece la coyuntura jurídica que permite oficialmente anclar una identidad afro en el espacio nacional, vale la pena decir que los esfuerzos de los afrodescendientes en Colombia por reclamar sus derechos tienen una larga trayectoria: desde tres puntos discursivos y geográficos que incluyen el interior, el Pacífico y la costa, el trabajo adelantado desde El Congreso, y la labor constante de intelectuales como los hermanos Zapata Olivella y su hermana Delia, y varios escritores afro previos. Cada uno aporta diferentes acercamientos a la lucha que convergen años más tarde en la constitución del 91, sin afirmar de ningún modo que la Ley 70 y el supuesto carácter inclusivo de la nueva constitución eran el objetivo central. Esa discusión queda en el tintero.
} 
la rebelión que se convierte en inspiración para Zapata Olivella: "En los Estados Unidos los negros lanzaron el desafío a los racistas, afirmando que constituían una raza de seres hermosos: 'Black is beautiful"' (770). Además de la negritud que comparte Zapata Olivella con los afroestadounidenses, la tri-etnicidad de Hughes añade otra conexión que los une y afianza su relación:

Langston Hughes se confesaba mulato-zambo, pero que hubiera preferido ser negro puro. En realidad en sus arterias confluían las tres sangres de la mayoría de los caribeños. Su padre fue un mulato, hijo de un judío traficante de esclavos. Su abuela materna, mestiza, descendía por línea directa de un indio cherokee. Su caudal africano le llegaba por las vertientes materna y paterna. Sus rasgos indios visibles en el rostro se acentuaban mucho más en su alma, luna llena que reflejaba los soles de todos sus ancestros. El cuerpo bajo y redondo recordaba a sus ascendientes bantúes. Cualquiera que lo hubiera tratado podía adivinar que su afabilidad le era connatural. Alguien de sus antepasados debió ser un cortesano africano acostumbrado a lidiar príncipes en remotos imperios. (Caja 47, Vol. II, 461)

Para Zapata Olivella, el axioma Harlem-Hughes-Negritud-África-Diáspora contienen una historicidad demarcada en un espacio negro, la figura del intelectual negro, y una herencia africana traducida a una tri-etnicidad en la diáspora que conecta los contrapuntos de una resistencia hemisférica. Política y artísticamente, Zapata Olivella se lanza con aún más ahínco a la lucha por los derechos de los afrodescendientes después de su estadía con Hughes, convencido de que los esfuerzos que ocurren en Harlem por avanzar la causa negra a través de la Asociación Universal de Desarrollo Negro y la Unión de Trabajadores de Color, con sede en Harlem, parte central del plan de Marcus Garvey para unir a todos los negros en EEUU, pueden dar resultado en Colombia y América Latina. No cabe duda que dichas asociaciones y rutas de liberación fueron fuentes de inspiración para Zapata Olivella que lo llevaron a tener un papel central en el Primer Congreso de la Cultura Negra de las Américas que tuvo lugar del 24-28 de agosto de 1977 en Cali, Colombia, "twenty-eight years after [Franz] Fanon's speech at the Second Congress of Negro Writers in 1959” (Ratcliff, 2008: 1). ${ }^{31}$ No es el único congreso en el que participa Zapata Olivella, pero es el primero que toma lugar en Colombia, por lo que se conoce como "El Congreso" (1). La actividad política de Zapata Olivella está marcada tanto por nuevas perspectivas y procesos de identidad afro, como por la necesidad de denunciar el racismo, la marginalidad y la situación de los negros en las Américas. Además, y algo que separa a Zapata Olivella de otros intelectuales afro, éste insiste en la importancia de incluir en la causa a los indígenas y mestizos. En el pensamiento zapataolivellano la negritud se convierte en "an ideology that allowed for the reconciliation of Afro-Latin American identities that were complicated by racial mixture" (Ratcliff : 5). ${ }^{32}$ Ratcliff discute la aplicación de Zapata Olivella del concepto de negritud como síntesis formulada por Senghor al panorama étnico latinoamericano donde la teoría del intelectual senegalés encontraría su más clara expresión (5). El trabajo de Zapata Olivella cuando hacía sus prácticas de medicina rural, su herencia tri-étnica, su vida en Lorica y en barrios entonces de clase trabajadora en Cartagena, y las infinitas injurias que

\footnotetext{
31 "veintiocho años después del discurso de [Franz] Fanon en el Second Congress of Negro Writers en 1959" (Traducción mía).

32 "una ideología que permitió la reconciliación de las identidades afrolatinoamericanas complicadas por la mezcla racial” (Traducción mía).
} 
sufre como profesional negro informan su propuesta de una negritud militante que pueda reconciliar, dice Ratcliff, negritud y mestizaje en el contexto latinoamericano y colombiano. La herencia tri-étnica de Hughes le da el sello de aprobación a la propuesta inclusiva que Zapata Olivella había ya formulado, con toda certidumbre. En El Congreso, Zapata Olivella y otros intelectuales "would meet to discuss their various interpretations of black solidarity with other Afro-descendant and African intellectuals in order to firmly inscribe Afro-Latin America into the Pan-African world" (5). ${ }^{33} \mathrm{El}$ transfondo de toda su actividad intelectual y política era esta premisa.

\section{Langston y Zapata Olivella en el continuo literario pan-afroamericano}

El concepto de un pan-afroamericanismo literario desarrollado por Christel Temple "represents a 'synthesis between history and literature,' in which authors share a 'desire to regenerate relationships, historical understandings and future interaction"' (Cit. en Ratcliff: 3). ${ }^{34}$ La idea de un compañerismo transnacional como el que existió entre Hughes y Zapata Olivella evoca un frente unido contra el racismo y un apoyo mutuo entre afrodescendientes en la Diáspora. Al compartir la misma posición ideológica, ambos intelectuales exhiben una consciencia pan-afroamericana que cuestiona y se esfuerza por cambiar los discursos nacionales supremacistas que perpetúan la opresión de los negros y marginados. Ratcliff ofrece el ejemplo de la relación entre Hughes y Nicolás Guillén, y menciona los estudios comparados de Miriam DeCosta-Willis, quien enfatiza el compromiso del último contra las políticas raciales transnacionales (3). Este axioma de cooperación y mutua comprensión entre intelectuales negros funciona igual en la relación Hughes y Zapata Olivella, afianzada dentro del legendario espacio diaspórico de Harlem. Así, no es solo el intercambio intelectual lo que une a los dos escritores, sino también su lucha por reclamar un espacio propio, al tiempo que navegan la realidad de las naciones y el hemisferio en que existen.

Hughes, escribe Zapata Olivella, le ofrece la estrategia para convertir la discriminación de la que eran víctimas en sus espacios correspondientes en las Américas en un arma construida por la música y la poesía:

La música y la poesía para nosotros [dice Hughes] es un arma. No importa cuál sea el tema de nuestra inspiración, lo importante es demostrar que un negro puede hacer cosas tan espirituales que muchos blancos con su poder económico, sus perros policías y sus mentiras no pueden alcanzar. (Zapata Olivella "Langston Hughes o el valor de la poesía" Caja 47, Vol. II, 458)

Ver el acto de la escritura y la producción artística de esa forma, hace pensar a Zapata Olivella sobre el papel del intelectual negro: "Sus palabras [de Hughes] dilucidando el papel del intelectual

\footnotetext{
33 "Se reunían para discutir sus diversas interpretaciones de la solidaridad negra con otros intelectuales afrodescendientes y africanos para inscribir firmemente a Afrolatinoamérica en el mundo panafricano" (5). (Traducción mía). Los números de página de las citas siguen la numeración de la versión digital del artículo de Ratcliff. Hay mucho más que considerar en los debates sobre los diferentes conceptos raciales que los líderes afro de la época. Muchos intelectuales, como el brasilero Abdias do Nascimento, rechazaban la idea de negritud como síntesis y condenaban el concepto de “'racial democracy' and mesticagem (racial mixing). Nascimento relates these concepts to the history of embranqueamento (whitening), which constitutes racial and cultural genocide against Africans in the Americas" (Ratcliff 5). Las citas que incluyo en este ensayo son parte de las fuentes de apoyo que he escogido y, sin duda, algunos elementos pueden ser explicados con mayor precisión. Para examinar las discusiones entabladas en el contexto de El Congreso, ver Valero (2020).

34 "Representa una 'síntesis entre historia y literatura' en la que los autores comparten un 'deseo de regenerar relaciones, entendimientos históricos e interacción futura"'. (Traducción mía).
} 
negro en su lucha contra el racismo me habían permitido descorrer el telón de las sutilezas, los engaños y la hipocresía de la discriminación en mi propia sociedad caribeña" (459). Para Zapata Olivella, cada palabra de Hughes en Harlem -porque la combinación Hughes-Harlem es lo que produce el impacto en el autor- lo lleva a la reconsideración de preceptos y experiencias que ya creía clara, a la auto-búsqueda de un "Nuevo Zapata Olivella" quien, como el Nuevo Negro de Harlem, emergiera revitalizado y listo para regresar a la batalla por la liberación de los negros y oprimidos en su país:

La promiscuidad con aquel infatigable combatiente por la libertad de su raza, comprometiendo la paz que podrían granjearle la venta de sus libros y su fama, desnudó mis sueños de vagabundo. La noche oscura de la alienación desaparecía con su presencia vitalizadora. El fuego de sus poemas depuraba mis confusiones, iluminaba mi extirpe de esclavo rebelde, las frustraciones de estudiante pobre, mi ideal de escritor. (460)

Estas líneas proyectan el embeleso de Zapata Olivella bajo la influencia de Hughes en "la estrechura y el calor de su apartamento en Harlem [...] un fortín de donde disparaban [Hughes y su tía, con quien compartía el pequeño apartamento] contra los depredadores de su pueblo" (459). La fortaleza intelectual e ideológica que construye Zapata Olivella después de su estadía en Harlem y con Hughes, le brinda el espacio para lanzar sus ataques, sus disparos ideológicos contenidos en novelas, obras de teatro, investigaciones culturales, crónicas, artículos de opinión, etc., a su vuelta a Colombia. Valdría explorar este punto más a fondo para trazar una cartografía de su producción intelectual que contribuyera a nivel tanto crítico como a nivel de praxis.

A manera de posdata, vale anotar que, a pesar del sinnúmero de contrapuntos que se pueden establecer dentro de la obra y el ideario zapataolivellanos con Harlem, en ninguna otra dimensión está mejor expresado lo que Harlem significaba para el autor que en el hecho de haber nombrado a una de sus hijas como tal: Harlem. Un imborrable testimonio de su conexión con dicho espacio.

\section{BIBLIOGRAFÍA}

Aldana, L. (2006). Entre mito e historia: hacia una estética fanoniana de la resistencia en "El árbol brujo de la libertad" de Manuel Zapata Olivella. Afro-Hispanic Review, 25(1), 39-53.

Appiah, A. (1985). The Uncompleted Argument: DuBois and the Illusion of Race. Critical Inquiry 12/1, pp. 21-37.

A United Statesian Celebrates.(Magazine Desk)(Letter to the editor). (1986). The New York Times Magazine.

Captain-Hidalgo, Y. (1993). The Culture of Fiction in the Works of Manuel Zapata Olivella. Columbia, Missouri: Missouri UP. 
DeCosta-Willis, M. (1973). Nicolás Guillén and His Poetry for Afro Americans. Black World 22/ (11), 12-16.

Ferguson, J. B. (2008). The Harlem Renaissance: A Brief History with Documents. Boston: Bedford/ San Martin.

Flóres Bolívar, F. J.( 2015). Un diálogo diaspórico: el lugar del Harlem Renaissance en el pensamiento racial e intelectual afrocolombiano (1920-1948). Historia crítica, (55), 101-124.

Hughes, L. (2002). Autobiography: The Big Sea. In: The Collected Works of Langston Hughes, Vol. 13. Joseph McLaren ed. Columbia, Missouri : Univ. of Missouri Press.

Hughes, L. "Harlem.” Poetry Foundation Recuperado de https:/www.poetryfoundation.org/ poems/46548/harlem

Hughes, L. (1958). The Langston Hughes Reader. New York: G. Braziller.

Jackson, R. (1981). The Shared Vision of Langston Hughes and Black Hispanic Writers. Black American Literature Forum, 15(3), 89-92.

Johnson, J. W. (1968). Black Manhattan. New York: Arno Press.

Leino, L. El legado que deja...Langston Hughes: Risas y lágrimas del negro. Manuel Zapata Olivella Collections, Jean and Alexander Heard Library, Vanderbilt University, Box 45.

Lomas, L. (2009). José Martí's “Evening of Emerson” and the United Statesian Literary Tradition. Journal of American Studies, 43(1), 1-17.

Mullen, E. (1977). Langston Hughes in the Hispanic World and Haiti. North Haven: Shoe String Press Inc..

Nascimento, A. (1979). Genocide: The Social Lynching of Africans and Their Descendants in Brazil. En: Brazil Mixture of Massacre (pp. 57-94). Dover, MA: The Majority Press.

Osofsky, G. (1966). Harlem, the Making of a Ghetto: Negro New York, 1890-1930. Harper \& Row.

Palacios, G. Manuel Zapata Olivella (1920-2004): pensador político, radical y hereje de la diáspora africana en las Américas. (2020). Medellín: Pontificia Universidad Bolivariana. 
Prescott, L. (2012). Liberating Blackness: The Theme of Whitening in Two Colombian Short Stories. Callaloo, 35(2), 475-493, 565.

Prescott, L. (2007). Journeying Through Jim Crow: Spanish American Travelers in the United States During the Age of Segregation. Latin American Research Review, 42(1), 3-28.

Prescott, L. (2006). Brother to Brother: The Friendship and Literary Correspondence of Manuel Zapata Olivella and Langston Hughes1. Afro-Hispanic Review, 25(1), 87-103, 252.

Proceedings of the Primer Congreso de la Cultura Negra de las Américas, UNESCO, 1988.

Ratcliff, A. (2008) "Black Writers of the World, Unite!"; Negotiating Pan-African Politics of Cultural Struggle in Afro-Latin America. The Black Scholar, 37(4), 27-38.

Saffire, W. (1986). On Language; Who Is an “American”? (Magazine Desk). The New York Times Magazine.

Temple, C. N. (2005). Literary Pan-Africanism: History, Contexts, and Criticism. Durham: Carolina Academic Press.

Valero, S. (2020). “Los negros se toman la palabra." Primer Congreso de la Cultura Negra de las Américas: Debates al interior de las comisiones y plenarias. Bogotá-Cartagena: Universidad Javeriana, Universidad de Cartagena, CEA.

Valero, S. y Campos García A. (Eds.) (2015). Identidades Políticas En Tiempos de Afrodescendencia: Auto-Identificación, Ancestralidad, Visibilidad y Derechos, Buenos Aires: Ediciones Corregidor.

Zapata Olivella, M. (2010). Harlem olvidado. Sábado [25 de diciembre, 1948]: 6-14. En: Manuel Zapata Olivella, por los senderos de sus ancestros: Textos escogidos 1940-2000. Vol. XVIII. Múnera A. (Ed.) (pp. 96-103). Bogotá: Biblioteca de Literatura Afrocolombiana.

Zapata Olivella, M. (1969). He visto la noche: las raíces de la furia negra. Medellín: Editorial Bedout.

Zapata Olivella, M. (1955). Hotel de vagabundos. Colombia: Ediciones Espiral.

Zapata Olivella, M. (6 de septiembre de1947). Miserias de New York. Sábado, 13, 15.

Zapata Olivella, M. Negritud y liberación. Manuel Zapata Olivella Collections, Jean and Alexander Heard Library, Vanderbilt University, Box47, Vol. III, pp.768-784. 
Zapata Olivella, M. Langston Hugues o el valor de la poesía. Manuel Zapata Olivella Collections, Jean and Alexander Heard Library, Vanderbilt University, Box 47, Vol II, 458-469.

Zapata Olivella, M. (2000). Pasión vagabunda. Colombia: Ministerio de Cultura. 